\title{
SIMPLE NEAR-RING CENTRALIZERS OF FINITE RINGS
}

\author{
CARLTON J. MAXSON AND KIRBY C. SMITH
}

\begin{abstract}
For a finite ring $R$ with identity and a finite unital $R$-module $V$ we call $C(R)=\{f: V \rightarrow V \mid f(\alpha v)=\alpha f(v)$ for all $\alpha \in R, v \in V\}$ the nearring centralizer of $R$. We investigate the structure of $C(R)$ and obtain a characterization of those rings $R$ for which $C(R)$ is a simple nonring.
\end{abstract}

1. Introduction. Let $H$ be a commutative ring with 1 and let $V$ be a finitely generated $H$-module with $S=\operatorname{End}_{H}(V)$. If $H$ is a field and if $R$ is a simple subring of $S$ containing $H$ then it is well known that the centralizer of $R$ in $S$, $C_{S}(R)$, is a simple ring. However if $C_{S}(R)$ is simple it is easy to see that $R$ need not be simple. As far as the authors know, a characterization of the subrings $R$ of $S$ such that $C_{S}(R)$ is simple is an unsolved problem. In this paper we consider a nonlinear analogue. In particular let $S$ denote the near-ring $S=\operatorname{Map}_{H}(V)=\{f: V \rightarrow V \mid f(\alpha v)=\alpha f(v)$ for all $\alpha \in H, v \in$ $V$ \}. Let $R$ be a subring of $S$ of $H$-endomorphisms of $V$ and assume $R$ contains $H$. The set $C(R)=\{f \in S \mid f(r v)=r f(v)$ for all $r \in R, v \in V\}$ is a near-ring, called the near-ring centralizer of $R$ in $S$. It is the goal of this paper to consider the following problems in the case where $H$ is a finite commutative ring with 1: If $R$ is a simple ring is $C(R)$ a simple near-ring? If $C(R)$ is a simple near-ring what can be said about $R$ ? Which simple nonrings (nearrings that are not rings) have the form $C(R)$ for some subring $R$ of $S$ ?

The above questions arise naturally from a different point of view. It has been shown by Betsch [1] that if $N$ is a finite simple near-ring which is not a ring then there exists a finite group $G$ and a fixed point free group of automorphisms $\Gamma$ of $G$ such that $N \cong C_{\Gamma}(G) \equiv\{f: G \rightarrow G \mid f(\gamma a)=\gamma f(a)$ for all $\gamma \in \Gamma, a \in G\}$. As long as $G$ is a group and $\Gamma$ is a semigroup of endomorphisms of $G$ then $C_{\Gamma}(G)$ forms a near-ring. The structure of $C_{\Gamma}(G)$ for various $G$ 's and $\Gamma$ 's has been investigated in [4], [5] and [6]. In this paper we study the structure of $C_{\Gamma}(G)$ when $\Gamma$ is a finite ring with 1 and $G$ is a unitary $\Gamma$-module. In this setting we investigate the problems mentioned above.

2. Simple centralizers. For the rest of this paper $R$ will denote a finite ring with 1 and $V$ a finite unital $R$-module. The near-ring centralizer of $R$ is $C(R)=\{f: V \rightarrow V \mid f(r v)=r f(v)$ for all $r \in R, v \in V\}$.

Presented to the Society, January 27, 1979; received by the editors June 26, 1978 and, in revised form, September 15, 1978.

AMS (MOS) subject classifications (1970). Primary 16A76, 16A44; Secondary 16A42, 16A48.

Key words and phrases. Centralizers, simple rings, near-rings. 
THEOREM 1. Let $R$ be a finite simple ring and let $V$ be a finite unital $R$-module. Then $C(R)$ is a simple near-ring. Moreover $C(R)$ is a nonring if and only if $R$ is a field and $\operatorname{dim}_{R} V>1$.

Proof. Let $\left\{e_{i j}\right\}$ be a set of matrix units for $R$ where $1=e_{11}+\cdots+e_{t t}$, the $e_{i i}$ primitive idempotents. The $R$-module $V$ forms a vector space over the center $F$ of $R$. We have $V=V_{1} \oplus \cdots \oplus V_{t}$ where $V_{i}=e_{i i} V$, a vector space direct sum. For $f \in C(R), v_{i} \in V_{i}$ we have $f\left(v_{1}+\cdots+v_{t}\right)=v_{1}^{\prime}+\cdots+$ $v_{t}^{\prime}$ for some $v_{i}^{\prime} \in V_{i}$. Since $e_{i i} f=f e_{i i}$ we have $f\left(v_{i}\right)=v_{i}^{\prime}$ and so $f\left(v_{1}+\cdots+\right.$ $\left.v_{t}\right)=f\left(v_{1}\right)+\cdots+f\left(v_{t}\right)$.

If $t=1$ then $R$ is a field and $C(R)$ is well known to be simple [1]. Also $C(R)$ is a nonring when $\operatorname{dim}_{R} V>1$. (For if $v \neq 0 \in V$ define $f: V \rightarrow V$ by $f(\alpha v)=\alpha v, \alpha \in R$, and $f(x)=0$ otherwise. Then $f \in C(R)$ and $f$ is not linear.)

Assume now that $t>1$. To show $f \in C(R)$ is a linear transformation on $V$ it suffices to show $f$ is linear on each $V_{i}$. Without loss of generality we choose $i=1$. For $v_{1} \in V_{1}, v_{2} \in V_{2}$ we have

$$
\begin{aligned}
\left(1+e_{12}\right) f\left(v_{1}+v_{2}\right) & =\left(1+e_{12}\right)\left(f\left(v_{1}\right)+f\left(v_{2}\right)\right) \\
& =f\left(v_{1}\right)+f\left(v_{2}\right)+f\left(e_{12} v_{2}\right),
\end{aligned}
$$

and

$$
\begin{aligned}
f\left(\left(1+e_{12}\right)\left(v_{1}+v_{2}\right)\right) & =f\left(v_{1}+v_{2}+e_{12} v_{2}\right) \\
& =f\left(v_{1}+e_{12} v_{2}\right)+f\left(v_{2}\right) .
\end{aligned}
$$

Hence $f\left(v_{1}+e_{12} v_{2}\right)=f\left(v_{1}\right)+f\left(e_{12} v_{2}\right)$. Since $e_{12} V_{2}=V_{1}, f$ is linear on $V_{1}$. Thus $C(R)$ is a ring and is simple by the double centralizer theorem for rings $[3$, p. 132].

COROllary. Assume $R$ is a finite semisimple ring and $V$ is a finite unital $R$-module. If none of the simple summands of $R$ is a field then $C(R)$ is a ring.

Assume now that $C(R)$ is a simple near-ring. From the Betsch characterization of simple near-rings [1] it is easy to see that the nonzero elements of the center of $C(R)$ are invertible. Since the center of $R$ is contained in the center of $C(R)$, the center of $R$ is a field $F$. Hence $R$ is an algebra over $F, V$ is a vector space over $F$, and $R$ may be viewed as a ring of linear transformations acting on $V$.

The Wedderburn principal theorem is valid for $R[7, \mathrm{p} .164]$, that is $R=S+N$, a vector space direct sum, where $N$ is the radical of $R$ and $S$ is semisimple with $R / N \cong S$.

If $N=(0)$ then $R$ must be simple since its center is a field, and Theorem 1 applies. Hence we may assume $N \neq(0)$. In the following sequence of lemmas we show that if $R$ is not a field then $C(R)$ is a ring.

Lemma 1. If $C(R)$ is simple with $R=S+N, N \neq(0)$, then $S$ is not a simple ring. 
Proof. Suppose $N^{k}=(0)$ with $N^{k-1} \neq(0)$. Let $W_{1}=\operatorname{ker} N^{k-1}=\{v \in$ $V \mid n v=0$ for all $\left.n \in N^{k-1}\right\}$, a proper subspace of $V$ and an $S$-submodule of $V$. As an $S$-module $V$ is completely reducible so $V=W_{1} \oplus W_{2}$, an $S$-module direct sum. Select $w_{2} \neq 0$ in $W_{2}$; then $S w_{2}$ is a direct summand of $W_{2}$ and $V=W_{1} \oplus S w_{2} \oplus \bar{W}_{2}$. The $S$-annihilator of $w_{2}$, $\operatorname{ann}\left(w_{2}\right)$, is a left ideal of $S$ so $S=\operatorname{ann}\left(w_{2}\right) \oplus S \bar{e}$ as left ideals, where $\bar{e}$ is an idempotent. If $\bar{e} N^{k-1} \neq(0)$ then there exists a $n_{0} \in N^{k-1}$ such that $\bar{e} n_{0} \neq 0$. Select $v \in V$ with $\bar{e} n_{0}(v) \neq$ 0 . Every element of $V$ can be uniquely written in the form $w_{1}+\bar{s} w_{2}+\bar{w}_{2}$. where $w_{1} \in W_{1}, \bar{s} \in S \bar{e}$ and $\bar{w}_{2} \in \bar{W}_{2}$. Define $f: V \rightarrow V$ by $f\left(w_{1}+\bar{s} w_{2}+\bar{w}_{2}\right)$ $=\overline{\operatorname{sen}} n_{0}(v)$. The function $f$ is nonzero since $f\left(\bar{e} w_{2}\right)=\bar{e} n_{0}(v) \neq 0$, and since $n W_{2} \subseteq W_{1}$ for all $n \in N$ we have $f \in C(R)$. Also the set $I=\{g \in$ $\left.C(R) \mid g\left(W_{1}\right)=\{0\}\right\}$ is an ideal of $C(R)$ containing $f$, which is a contradiction to the simplicity of $C(R)$, so $\bar{e} N^{k-1}=(0)$. Thus the $S$-annihilator of $N^{k-1}$ is a proper ideal of $S$.

LEMMA 2. Assume $C(R)$ is simple and not a ring. Then $S$ is a direct sum of fields.

Proof. We have $R=S+N$ where $S=S_{1} \oplus S_{2} \oplus \cdots \oplus S_{t}$, $S_{i}$ sinple and $t>1$. Suppose $S_{1}$ is not a field. Let $e_{i}$ be the identity for $S_{i}$. We have $V=e_{1} V \oplus \cdots \oplus e_{t} V$ where each $e_{i} V$ is $C(R)$-invariant. Since the simple ring $S_{1}$ acts on $e_{1} V$ the map $\Phi: C(R) \rightarrow C\left(S_{1}\right)$ given by $f \rightarrow \bar{f}$, where $\bar{f}$ is $f$ restricted to $e_{1} V$, is an imbedding. Since $C\left(S_{1}\right)$ is a ring, so is $C(R)$. Thus $S$ must be a direct sum of fields.

LEMMA 3. Suppose $C(R)$ is simple and that in $R, 1=e_{1}+e_{2}$ where $e_{1}$ and $e_{2}$ are orthogonal idempotents that belong to the center of $S$. Let $V=W_{1} \oplus W_{2}$ where $W_{i}=e_{i} V$. Assume there exists a positive integer l such that ker $N^{l} \subseteq W_{1}$ and $\operatorname{ker} N^{l+1} \cap W_{2} \neq(0)$. Then $C(R)$ is a ring.

Proof. Let $K=\operatorname{ker} N^{l+1} \cap W_{2}$, an $S$-submodule of $V$ which is $C(R)$ invariant. The subspace $\left\langle N^{l} K\right\rangle$ of $V$ is also an $S$-submodule, and $\left\langle N^{l} K\right\rangle \subseteq$ ker $N \subseteq \operatorname{ker} N^{l} \subseteq W_{1}$. As $S$-modules we have $V=\bar{W}_{1} \oplus\left\langle N^{l} K\right\rangle \oplus K \oplus \bar{W}_{2}$ where $W_{1}=\bar{W}_{1} \oplus\left\langle N^{l} K\right\rangle, W_{2}=K \oplus \bar{W}_{2}$.

We show now that if $f \in C(R)$ then $f$ is linear on $\left\langle N^{l} K\right\rangle \oplus K$. For $v_{1}, v_{2} \in K, n_{1}, n_{2} \in N^{l}$ we have $n_{i} v_{i} \in\left\langle N^{l} K\right\rangle$. Also $\left(1+n_{1}\right) f\left(v_{1}+n_{2} v_{2}\right)=$ $f\left(\left(1+n_{1}\right)\left(v_{1}+n_{2} v_{2}\right)\right)$ which implies that $f\left(n_{1} v_{1}+n_{2} v_{2}\right)=f\left(n_{1} v_{1}\right)+f\left(n_{2} v_{2}\right)$. For $n \in N^{l}, v_{1}, v_{2} \in K$ we have, from above, that $n\left(f\left(v_{1}+v_{2}\right)-f\left(v_{1}\right)-\right.$ $\left.f\left(v_{2}\right)\right)=0$. This means $f\left(v_{1}+v_{2}\right)-f\left(v_{1}\right)-f\left(v_{2}\right) \in \operatorname{ker} N^{l} \cap W_{2}=(0)$ and so $f$ is linear on $K$.

For $n_{i} \in N^{l}, v_{i} \in K, i=1,2,3$, we have

$$
\left(1+n_{1}\right) f\left(v_{1}+n_{2} v_{2}+n_{3} v_{3}\right)=f\left(\left(1+n_{1}\right)\left(v_{1}+n_{2} v_{2}+n_{3} v_{3}\right)\right)
$$

so

$$
f\left(n_{1} v_{1}+n_{2} v_{2}+n_{3} v_{3}\right)=f\left(n_{1} v_{1}\right)+f\left(n_{2} v_{2}\right)+f\left(n_{3} v_{3}\right)
$$


Continuing we get $f$ is linear on $\left\langle N^{l} K\right\rangle$, and hence linear on $\left\langle N^{l} K\right\rangle \oplus K$. Moreover since $K$ is $C(R)$-invariant so is $\left\langle N^{l} K\right\rangle$ and hence $\left\langle N^{l} K\right\rangle \oplus K$ is both $C(R)$ and $S$-invariant. Let

$$
\begin{array}{r}
C\left(S ;\left\langle N^{l} K\right\rangle \oplus K\right)=\left\{f:\left\langle N^{l} K\right\rangle \oplus K \rightarrow\left\langle N^{l} K\right\rangle \oplus K \mid f(\alpha v)=\alpha f(v),\right. \\
\left.\alpha \in S, v \in\left\langle N^{l} K\right\rangle \oplus K\right\} .
\end{array}
$$

The map given by $f \rightarrow \bar{f}$, where $\bar{f}$ is $f$ restricted to $\left\langle N^{\prime} K\right\rangle \oplus K$, is an imbedding of $C(R)$ into $C\left(S ;\left\langle N^{l} K\right\rangle \oplus K\right)$. Since the range of this imbedding is a ring, $C(R)$ is also a ring.

THEOREM 2. Suppose $R$ is a finite ring with 1 , not a field, and $V$ is a finite unital $R$-module. If $C(R)$ is simple then it is a ring.

Proof. From Lemmas 1 and 2 we have $R=S+N$ where $S$ is a direct sum of at least two fields and $N^{k}=(0), N^{k-1} \neq(0)$. So $S=S e_{1} \oplus \cdots \oplus$ $S e_{t}$ where each $e_{i}$ is a primitive idempotent, and $V=W_{1} \oplus \cdots \oplus W_{t}$ where $W_{i}=e_{i} V$.

As $S$-modules we have $V=\operatorname{ker} N^{k-1} \oplus \bar{W}$. If $\bar{w} \neq 0 \in \bar{W}$ then $\bar{w}=w_{1}$ $+\cdots+w_{t}, w_{i} \in W_{i}$, and we may assume $w_{t} \neq 0$. Then $e_{t} \bar{w}=w_{t} \in \bar{W} \cap$ $W_{t}$. We show now that ker $N \cap W_{t}=(0)$. Assume $k \neq 0$ belongs to $\operatorname{ker} N \cap$ $W_{t}$. As $S$-modules

$$
V=W_{1} \oplus W_{2} \oplus \cdots \oplus W_{t-1} \oplus \bar{S} w_{t} \oplus \bar{W}_{t}
$$

where $\bar{S}=S e_{t}$ and $W_{t}=\bar{S}_{w_{t}} \oplus \bar{W}_{t}$. Define $f: V \rightarrow V$ by

$$
f\left(w_{1}+w_{2}+\cdots+w_{t-1}+\bar{s} w_{t}+\bar{w}_{t}\right)=\bar{s} k .
$$

Since $n V \subseteq \operatorname{ker} N^{k-1}$ for all $n \in N$ and since $\bar{S} w_{t} \cap \operatorname{ker} N^{k-1}=(0)$, we have $f \in C(R)$. Also $f \neq 0$ since $f\left(w_{t}\right)=e_{t} k=k \neq 0$. Moreover $f\left(\operatorname{ker} N^{k-1}\right)=$ $(0)$ and this contradicts the simplicity of $C(R)$. Hence ker $N \cap W_{t}=(0)$.

We now have ker $N \subseteq W_{1} \oplus W_{2} \oplus \cdots \oplus W_{t-1}$ with $\operatorname{ker} N \cap W_{t}=(0)$. Let $l \geqslant 1$ be such that $\operatorname{ker} N^{l} \subseteq W_{1} \oplus \cdots \oplus W_{t-1}$ but $\operatorname{ker} N^{l+1} \& W_{1}$ $\oplus \cdots \oplus W_{t-1}$. Then there exists a $v \in \operatorname{ker} N^{l+1}$ with $v=w_{1}+\cdots+$ $w_{t-1}+w_{t}$ and $w_{t} \neq 0$. This means $e_{t} v=w_{t}$ is a nonzero element in ker $N^{t+1}$ $\cap W_{t}$. By Lemma 3, $C(R)$ is a ring.

Under the conditions of the above theorem, the simple near-ring $C(R)$ is a simple ring which clearly contains $\operatorname{End}_{R}(V)$, the ring of $R$-endomorphisms of $V$. We now show that $C(R)$ is in fact equal to the ring $\operatorname{End}_{R}(V)$. This is established by showing that the near-ring module $V$ is indeed a ring module over $C(R)$.

THeOREM 3. Suppose $R$ is a finite ring with 1 , not a field, and $V$ is a finite unital $R$-module. If $C(R)$ is simple then $C(R)=\operatorname{End}_{R}(V)$, the ring of $R$ endomorphisms of $V$.

Proof. From Theorem $2 C(R)$ is a ring. We need to show $C(R)=$ $\operatorname{End}_{R}(V)$. Clearly $V$ is a near-ring module over the simple ring $C(R)$. Since 
$C(R)$ is a ring it is a distributively generated near-ring, so submodules of $V$ and $C(R)$-subgroups of $V$ coincide [8, p. 174]. We have $C(R)=A_{1}$ $\oplus \cdots \oplus A_{t}$ where the $A_{i}$ are minimal left ideals of $C(R)$. For $v \in V$ either $A_{i} \cong A_{i} v$ or $A_{i} v=(0)$. Hence $V=\sum_{v \in V} \sum_{i} A_{i} v$, a sum of irreducible submodules of $V$. But then $V$ is a direct sum of irreducible submodules $V=V_{1} \oplus \cdots \oplus V_{s}$. Since each $V_{i}$ is a cyclic submodule of $V$ it is clear that $f \in C(R)$ is additive on each $V_{i}$. Using the definition of a near-ring module it is easy to see that $f \in C(R)$ is additive on all of $V$. Thus $V$ is a ring module and $C(R)$ is the complete ring of $R$-endomorphisms of $V$ as desired.

\section{REFERENCES}

1. G. Betsch, Some structure theorems on 2-primitive near-rings, Rings, Modules and Radicals (Proc. Colloq., Keszthely, 1971), Colloq. Math. Soc. János Bolyai, Vol. 6, North-Holland, Amsterdam, 1973, pp. 73-102.

2. I. N. Herstein, Noncommutative rings, Carus Monograph No. 15, Math. Assoc. of America, Washington, D. C., 1968.

3. N. Jacobson, The structure of rings, Amer. Math. Soc. Colloq. Publ., vol. 37, Amer. Math. Soc., Providence, R. I., 1964.

4. C. J. Maxson and K. C. Smith, The centralizer of a group automorphism, J. Algebra 54 (1978), $27-41$.

5.

6. $\ldots$ The centralizer of a set of group automorphisms (preprint).

7. B. R. McDonald, Finite rings with identity, Dekker, New York, 1974.

8. G. Pilz, Near-rings, North-Holland, Amsterdam, 1977.

Department of Mathematics, Texas A\&M University, College Station, Texas 77843 